\title{
The Vibration Analysis of Micro-chip for the Specific Heart Rates Population Detection with a Mechanical Robotic Manipulator
}

\author{
Jieyi Zhu ${ }^{1,2,3}$, Zuxiang Lei ${ }^{2, *}$ and Changan Zhu ${ }^{1}$ \\ ${ }^{1}$ Department of Precision Machinery and Precision Instrumentation, USTC, China \\ ${ }^{2}$ City University of HongKong, China \\ ${ }^{3}$ Institute of Intelligent Machines, Chinese Academy of Sciences, Hefei, China \\ azhujieyi@mail.ustc.edu.cn, ${ }^{b}$ leizux@mail.ustc.edu.cn, ${ }^{c}$ changan@ustc.edu.cn \\ * please mark the corresponding author with an asterisk
}

\begin{abstract}
Keywords: Mechanical Robotic Manipulator; Vibration Analysis; Specific Heart Rates detection Abstract. This paper presents a system design of mechanical robotic manipulator with its own sensor end effectors. For the end effectors, they would be fixed with a fabricated micro-nano sensor. This system's application is to detect specific heart rates of various population by executing a robotic arm action to measure people's radial signals. So the paper includes the mechanical modeling of the robotic manipulator, the design of sensor surface, the material vibration analysis, and the vibration signal process.
\end{abstract}

\section{Introduction}

Mechanical robotic arm has been widely studied and used in many years due to its high performance in industrial applications fields. The application fields include route planning, snatching objects, spot welding, and so on. Different mechanical robotic arms are designed with various modeling methods. For example, D-H(Denavit-Hartenburg) notation is to describe the translation and rotation relationship between each stick in one system[1]. This notation method build up a $4 \times 4$ homogeneous transformation matrix, which can represent the relationship between this stick and the other stick. In our project, the design of mechanical robotic arm is used in specific heart rates detection of the target persons

As heart rates of human beings is associated with radial vascular's vibration, we could use a vibration sensor to realize the detection of specific heart rates. In order to solve the problem of pulse wave detection, an robotic manipulator with its sensor end effectors system is designed. Robotic manipulator has proven to be a powerful tool for realizing target tracking. The piezoelectric sheet vibration simulation is done by Ansys software and discussed for the rectangular hole fabrication, due to the vibration mode and frequency calculation method[2] .The schematic diagram of the robotic manipulator is shown in Fig .1:

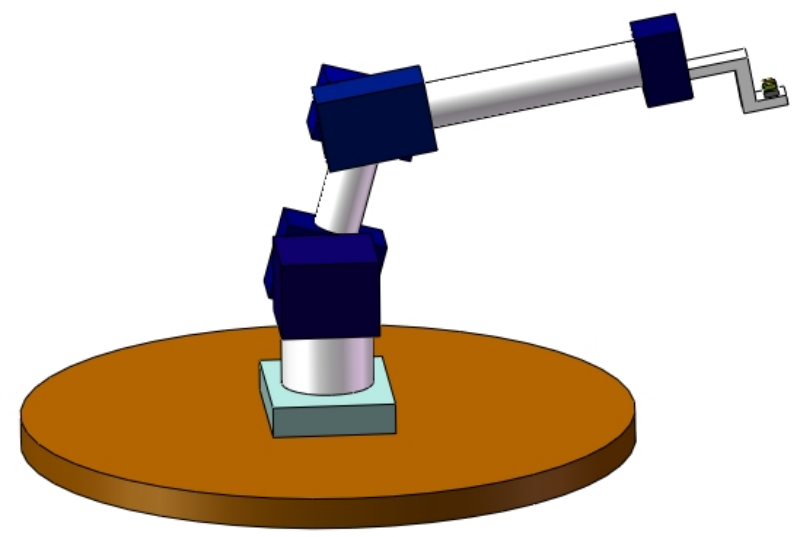

Fig .1 The schematic diagram of our design of robotic manipulator with its sensor end effectors 
In this paper's latter part, the mechanical analysis of material vibration is discussed. The Ansys software could help us calculate the sensor material's intrinsic frequency. By coupling the one vibration frequency with one certain heart rate of radial vascular pulse wave signal, we could use this vibration sensor to resolve for the corresponding heart rates. Thus, this kind of vibration sensor would be applicable in future medical use.

\section{Robotic Manipulator Modelling}

From the schematic design shown in Fig .1, we could simplify it into a position mathematical model for the mechanical robot manipulator. The simplified model would be a planar two-degree of freedom robotic arm model. The simplified model figure is shown in Fig .2:

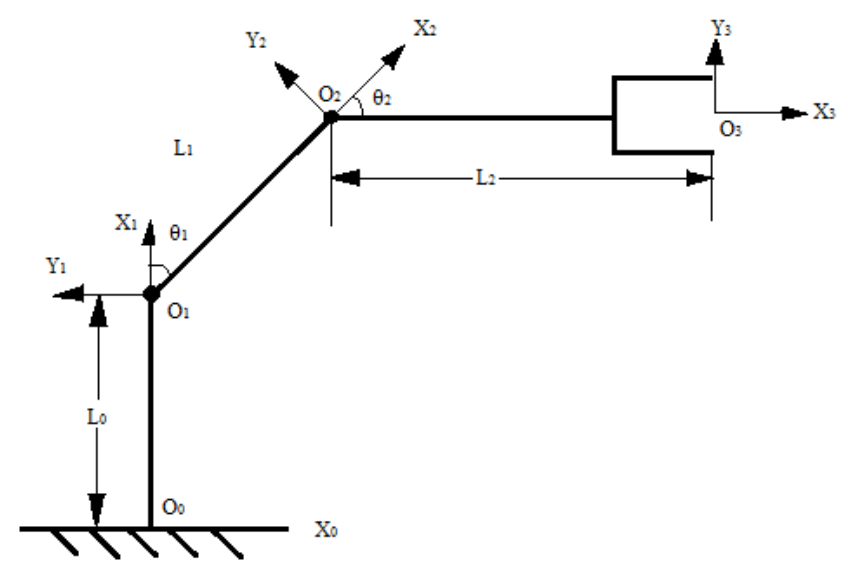

Fig. 2. The modeling of our manipulator in two degree of freedom design

For the modeling of the manipulator, a modeling method called D-H(Denavit-Hartenburg) notation is used[3]. In our system, there are three joints, the first one joint $\mathrm{O}_{0}$ is a fixed joint, the other two joints $\mathrm{O}_{1}$ and $\mathrm{O}_{2}$ are flexible joints. As shown in Fig .2, each stick's D-H parameter and joint variable are shown in Table.1:

\begin{tabular}{|c|c|c|c|}
\hline joint & $\mathrm{a}_{\mathrm{i}}$ & $\theta_{\mathrm{i}}$ & Joint variable \\
\hline $\mathrm{O}_{0}$ & $\mathrm{~L}_{0}$ & $90^{\circ}$ & \\
\hline $\mathrm{O}_{1}$ & $\mathrm{~L}_{1}$ & $\theta_{1}$ & $\theta_{1}$ \\
\hline $\mathrm{O}_{2}$ & $\mathrm{~L}_{2}$ & $\theta_{2}$ & $\theta_{2}$ \\
\hline
\end{tabular}

Table. 1. The D-H parameters and joint variable of each joint

Thus, the position matrix of our two degree of freedom series connection robotic arm is calculated as:

$$
{ }^{0} \mathbf{T}_{2}=\mathbf{A}_{0} \cdot{ }^{0} \mathbf{A}_{1} \cdot{ }^{1} \mathbf{A}_{2}
$$

The coordinate system transformational matrix of each stick is shown as below:

$$
\begin{aligned}
\mathbf{A}_{0}=\operatorname{Rot}\left(\mathrm{O}_{0}, 90^{\circ}\right) \cdot \operatorname{Trans}\left(\mathrm{X}_{0}, \mathrm{~L}_{0}\right) & =\left[\begin{array}{cccc}
0 & -1 & 0 & 0 \\
1 & 0 & 0 & 0 \\
0 & 0 & 1 & 0 \\
0 & 0 & 0 & 1
\end{array}\right] \cdot\left[\begin{array}{llll}
1 & 0 & 0 & L_{0} \\
0 & 1 & 0 & 0 \\
0 & 0 & 1 & 0 \\
0 & 0 & 0 & 1
\end{array}\right] \\
& =\left[\begin{array}{cccc}
0 & -1 & 0 & 0 \\
1 & 0 & 0 & L_{0} \\
0 & 0 & 1 & 0 \\
0 & 0 & 0 & 1
\end{array}\right] \\
{ }^{0} \mathbf{A}_{1}=\operatorname{Rot}\left(O_{1}, \theta_{1}\right) \cdot \operatorname{Trans}\left(X_{1}, L_{1}\right)= & {\left[\begin{array}{cccc}
\cos \theta_{1} & -\sin \theta_{1} & 0 & 0 \\
\sin \theta_{1} & \cos \theta_{1} & 0 & 0 \\
0 & 0 & 1 & 0 \\
0 & 0 & 0 & 1
\end{array}\right] \cdot\left[\begin{array}{llll}
1 & 0 & 0 & L_{1} \\
0 & 1 & 0 & 0 \\
0 & 0 & 1 & 0 \\
0 & 0 & 0 & 1
\end{array}\right] }
\end{aligned}
$$




$$
\begin{aligned}
& =\left[\begin{array}{cccc}
\cos \theta_{1} & -\sin \theta_{1} & 0 & L_{1} \cos \theta_{1} \\
\sin \theta_{1} & \cos \theta_{1} & 0 & L_{1} \sin \theta_{1} \\
0 & 0 & 1 & 0 \\
0 & 0 & 0 & 1
\end{array}\right] \\
{ }^{1} \mathbf{A}_{2}=\operatorname{Rot}\left(O_{2}, \theta_{2}\right) \cdot \operatorname{Trans}\left(X_{2}, L_{2}\right) & {\left[\begin{array}{ccccc}
\cos \theta_{2} & -\sin \theta_{2} & 0 & 0 \\
\sin \theta_{2} & \cos \theta_{2} & 0 & 0 \\
0 & 0 & 1 & 0 \\
0 & 0 & 0 & 1
\end{array}\right] \cdot\left[\begin{array}{cccc}
1 & 0 & 0 & L_{2} \\
0 & 1 & 0 & 0 \\
0 & 0 & 1 & 0 \\
0 & 0 & 0 & 1
\end{array}\right] } \\
& =\left[\begin{array}{cccc}
\cos \theta_{2} & -\sin \theta_{2} & 0 & L_{2} \cos \theta_{2} \\
\sin \theta_{2} & \cos \theta_{2} & 0 & L_{2} \cos \theta_{2} \\
0 & 0 & 1 & 0 \\
0 & 0 & 0 & 1
\end{array}\right]
\end{aligned}
$$

Thus, the position matrix is calculated as

$$
{ }^{0} \mathbf{T}_{\mathbf{2}}=\mathbf{A}_{\mathbf{0}} \cdot{ }^{0} \mathbf{A}_{\mathbf{1}} \cdot{ }^{\mathbf{1}} \mathbf{A}_{\mathbf{2}}=\left[\begin{array}{cccc}
-\sin \left(\theta_{1}+\theta_{2}\right) & -\cos \left(\theta_{1}+\theta_{2}\right) & 0 & -L_{2} \sin \left(\theta_{1}+\theta_{2}\right)-L_{1} \sin \theta_{1} \\
\cos \left(\theta_{1}+\theta_{2}\right) & -\sin \left(\theta_{1}+\theta_{2}\right) & 0 & L_{2}\left(\theta_{1}+\theta_{2}\right)+L_{0}+L_{1} \cos \theta_{1} \\
0 & 0 & 1 & 0 \\
0 & 0 & 0 & 1
\end{array}\right]
$$

\section{Sensor Material Analysis}

In order to analyze the sensor performance, we need to conduct calculation and simulation for the sensor material. For our chosen sensor material piezoelectric ceramic, it not only has excellent electrical property, but also share outstanding mechanical property. In our designed sensor surface, the mechanical model is a piezoelectric ceramic rectangular sheet with clamped fixing for the peripheral carbon atoms. As the flowing gas to be detected could cause the piezoelectric ceramic sheet vibration, so the calculation of piezoelectric ceramic vibration would be meaningful. For the first of all, we need to prepare material properties of piezoelectric ceramic sheet, the overall parameters for calculation is listed in Table. 2:

\begin{tabular}{c|c}
\hline Young's Modulus(E) & $76.5 \mathrm{GPa}$ \\
\hline Poisson Ratio(v) & 0.32 \\
\hline Density $(\rho)$ & $7500 \mathrm{Kg} / \mathrm{m}^{3}$ \\
\hline Thickness(h) & $0.335 \mathrm{~nm}$ \\
\hline
\end{tabular}

Table.2 Piezoelectric ceramic Sheet Material Properties

From the micro-nano fabrication, we could see that our substrate has one rectangular hole in the middle, and the size of the rectangular hole could be changed during our one fabrication step. So after fabrication our sensor surface structure is shown in Fig. 3:

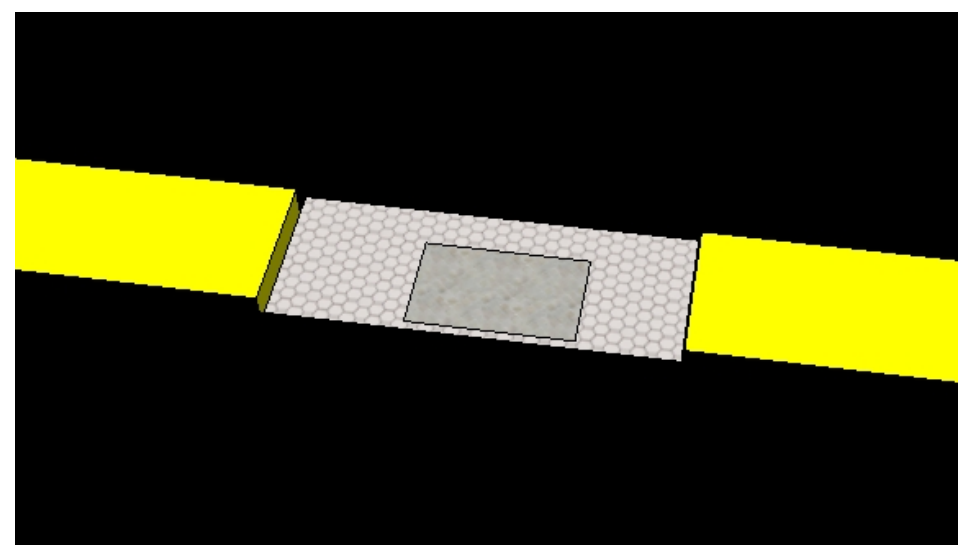

Fig .3 Schematic of the sensor surface structure, the substrate is with one rectangular hole in the middle.

From the sensor material discussion, we could use Ansys to process the piezoelectric ceramic sheet vibration calculation. In Ansys software, the piezoelectric ceramic sheet is processed as one rectangular plate[4].In the Ansys model analysis, the important piezoelectric ceramic parameters are considered, including Young's Modulus(E), Poisson Ratio, density( $\rho)$, and thickness(h). The Ansys 
analysis and calculation are based on Finite Element Modelling(FEM), which is one common calculation method for vibration frequency calculation[5]. In our simulation, the piezoelectric ceramic sheet is set to four groups of sizes, including $10 \mathrm{~nm} \times 10 \mathrm{~nm}, 20 \mathrm{~nm} \times 20 \mathrm{~nm}, 50 \mathrm{~nm} \times 50 \mathrm{~nm}, 100 \mathrm{~nm} \times 100 \mathrm{~nm}$. The piezoelectric ceramic plate is divided into smaller meshes by the Ansys mesh function, in our simulation we choose quadrangle meshes for the piezoelectric ceramic plate. The Ansys meshing figure is shown in Fig.4.

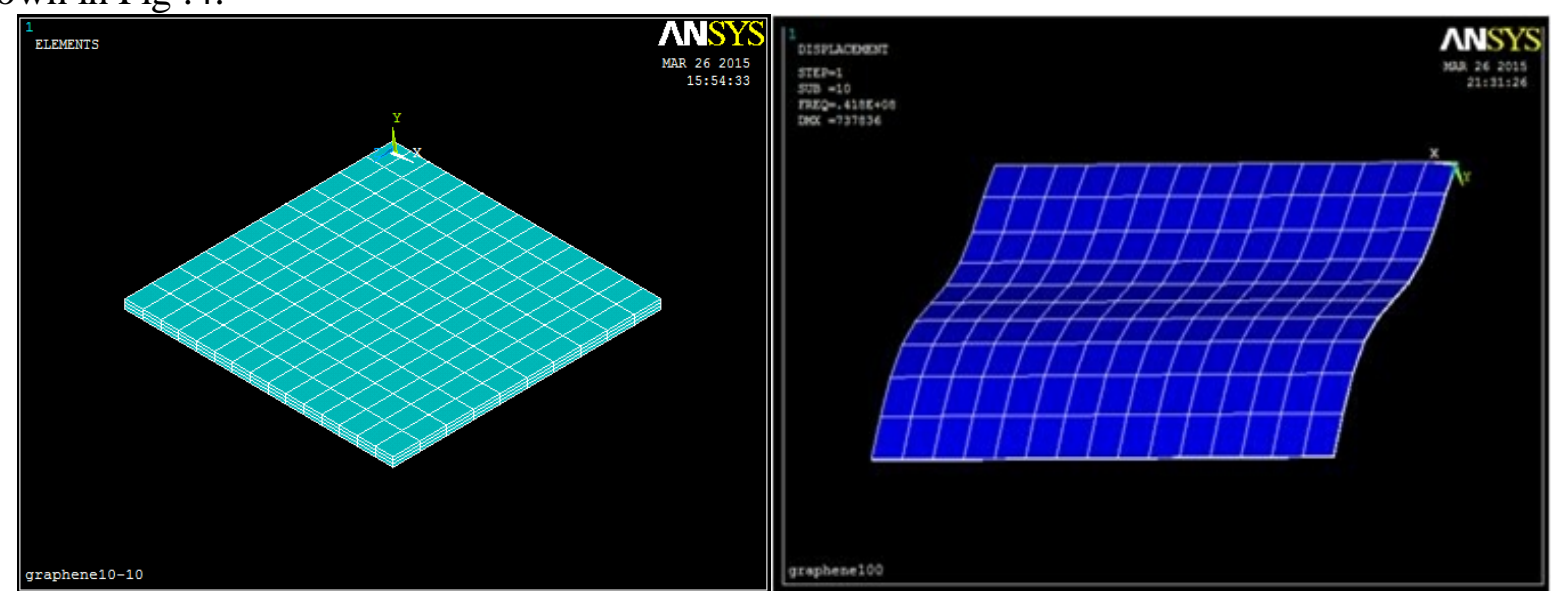

Fig .4. Finite element process for Piezoelectric ceramic sheet model in Ansys software. The Piezoelectric ceramic sheet size in figure is $10 \mathrm{~nm} \times 10 \mathrm{~nm}$.

For the calculation process, it's based on Kirchhoff plate theory[6]. In Ansys simulation, the larger Piezoelectric ceramic plate is divided into many smaller rectangular quadrangle meshes. In each small mesh, the displacement in both directions are:

$$
\left\{\begin{array}{l}
U=-z \times \alpha_{x} \\
V=-z \times \alpha_{y} \\
Z=-z \times \alpha_{z}
\end{array}\right.
$$

And the strain/displacement equations are:

$$
\left\{\begin{array}{l}
\varepsilon_{x}=-\frac{\partial^{2} w}{\partial x^{2}} \\
\varepsilon_{y}=-\frac{\partial^{2} w}{\partial y^{2}} \\
\varepsilon_{z}=-\frac{\partial^{2} w}{\partial z^{2}}
\end{array}\right.
$$

The plane stress equations for the piezoelectric ceramic material are:

$$
\left\{\begin{array}{l}
\sigma_{x}=\frac{E}{1-\theta^{2}}\left(\varepsilon_{x}+\theta \varepsilon_{y}\right) \\
\sigma_{y}=\frac{E}{1-\theta^{2}}\left(\varepsilon_{y}+\theta \varepsilon_{x}\right)
\end{array}\right.
$$

According to Kirchhoff theory,bending moments are:

$$
\left\{\begin{array}{l}
M_{x}=\int_{-h / 2}^{h / 2} z * \sigma_{x} d z \\
M_{y}=\int_{-h / 2}^{h / 2} z * \sigma_{y} d z
\end{array}\right.
$$

For the stiffness matrix calculation, for the isotropic case:

$$
[k]=\frac{E h^{3}}{48\left(1-\theta^{2}\right) a b}\left[\begin{array}{llll}
k_{11} & k_{12} & k_{13} & k_{14} \\
k_{21} & k_{22} & k_{23} & k_{24} \\
k_{31} & k_{32} & k_{33} & k_{34} \\
k_{41} & k_{42} & k_{43} & k_{44}
\end{array}\right]
$$


For the overall calculation, the element stiffness and mass matrices are assembled for the eigenvalue and frequency. The equation of motion of the plate can be writen as[7]:

$$
[k]-\omega^{2}[M]=0
$$

From which the eigenvalues $(\omega)$ can be extracted. The smallest value of $\omega$ is the fundamental natural frequency[8]. For the result, we conduct modal analysis in the Ansys 14.0 software, the resonant mode is picked out for $10 \mathrm{~nm} \times 10 \mathrm{~nm}, 20 \mathrm{~nm} \times 20 \mathrm{~nm}, 50 \mathrm{~nm} \times 50 \mathrm{~nm}, 100 \mathrm{~nm} \times 100 \mathrm{~nm}$ Piezoelectric ceramic sheet respectively. And the resonant frequency values are listed in the Table.3.

\begin{tabular}{c|c}
\hline $\begin{array}{c}\text { Piezoelectric ceramic sheet } \\
\text { size }\end{array}$ & Resonant frequency \\
\hline $10 \mathrm{~nm} \times 10 \mathrm{~nm}$ & $5.8825 \times 10^{10} \mathrm{~Hz}$ \\
\hline $20 \mathrm{~nm} \times 20 \mathrm{~nm}$ & $2.2175 \times 10^{10} \mathrm{~Hz}$ \\
\hline $50 \mathrm{~nm} \times 50 \mathrm{~nm}$ & $2.8775 \times 10^{9} \mathrm{~Hz}$ \\
\hline $100 \mathrm{~nm} \times 100 \mathrm{~nm}$ & $2.1419 \times 10^{9} \mathrm{~Hz}$ \\
\hline
\end{tabular}

Table3. Resonant frequencies of four Piezoelectric ceramic sheets

From the calculation results above, we choose the $100 \mathrm{~nm} \times 100 \mathrm{~nm}$ size for the optimization of vibration signal analysis.

\section{Vibration Signal Process}

In our medical experiment, we acquire patients' radial waveform signals during our clinical measurement. Among our patients, we collected different heart rates' patients, including low heart rates $(<75)$, and high heart rates $(>90)$. The schematic diagram of one paradigmatic radial waveform of a 70 beats/min heart rate patient is shown in Fig. 5:

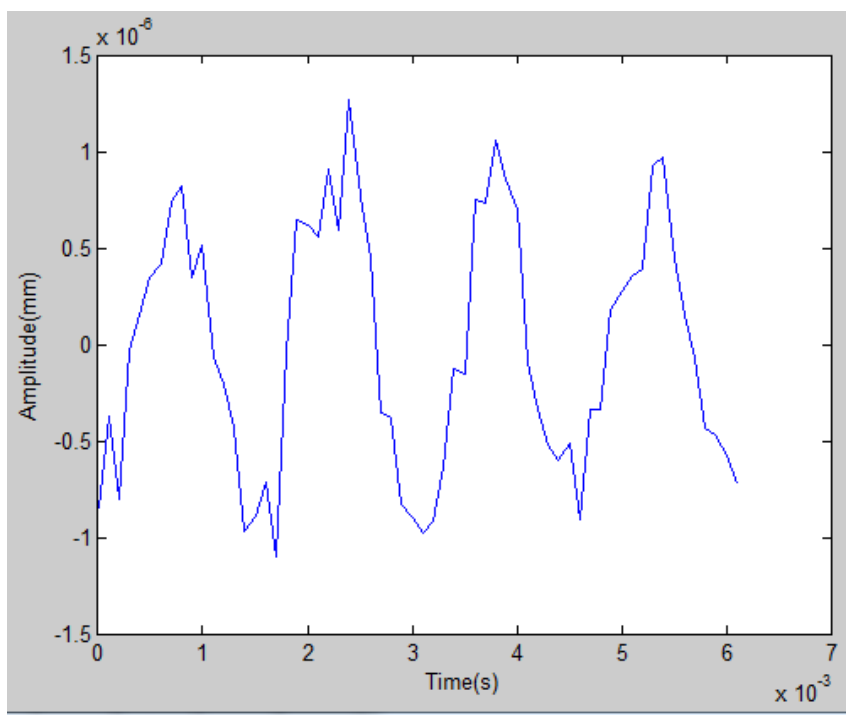

Fig .5 The schematic radial waveform of a 70 beats/min heart rates patient.

For our matlab radial signal processing, we record different heart rates' patients' radial waveform signals, and use our vibration sensor to attach on those patients' left radial artery of the left arm. All measurement were performed in air-conditioned room $\left(22-26^{\circ} \mathrm{C}\right)$. Each subject rested for at least 10 mins before measurements[9]. During our experiment, we used two patients' waveform signals as the two examples, which is shown in Fig. 6. In the left figure of Fig. 6, we recorded a low heart rate patient, whose heart rate is about 60. In the right figure of Fig. 6, we recorded a high heart rate patient, whose heart rate is 88 . And after our detection by using the mentioned vibration sensor, the recorded resonant frequency is shown in Fig. 6, the low heart rate patient is corresponding to a lower resonant frequency, while the high heart rate patient is corresponding to a higher resonant frequency. Thus, after more time's experiments, we could find a whole relationship between heart rate and resonant frequency recorded by the vibration sensor. 


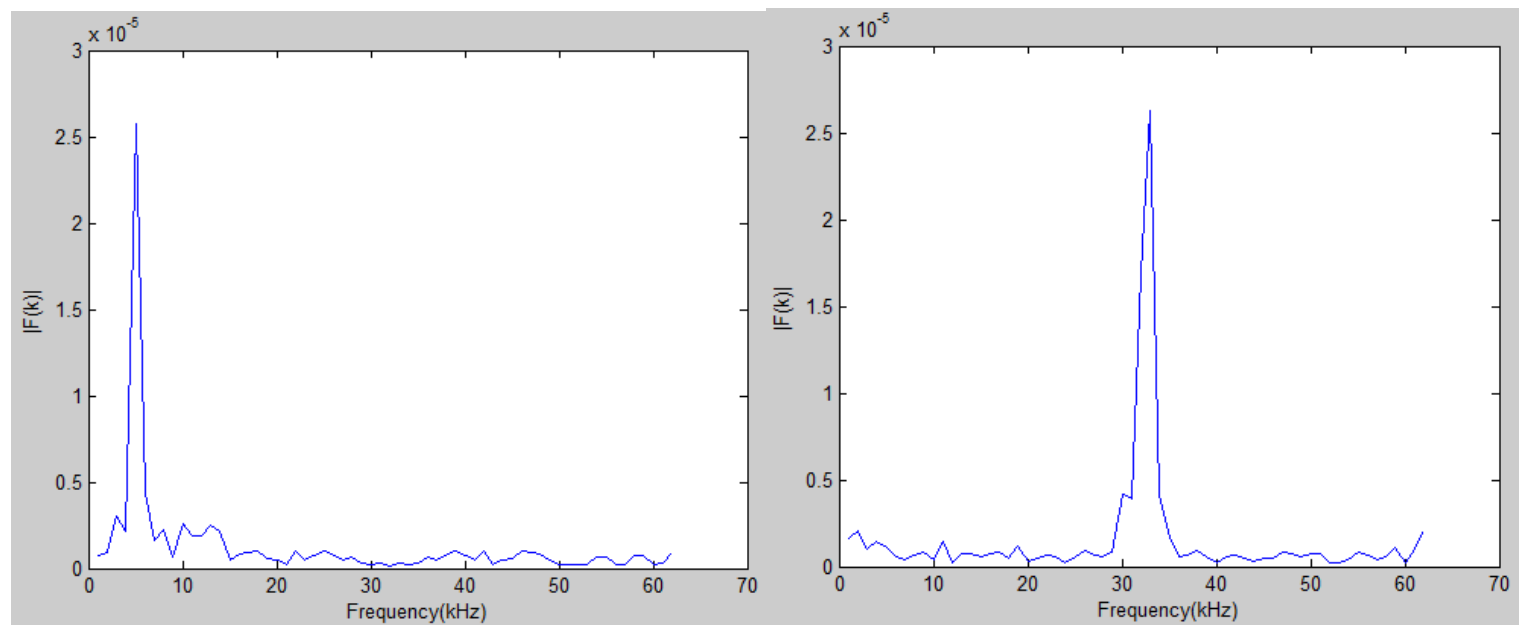

Fig .6 The matlab correlation for low heart rates and high heart rates patients by the detection of our vibration sensor

\section{Conclusion}

In conclusion, we designed a robot manipulator with a heart rate sensor on the end effector.We conduct modelling of the robotic manipulator, and fabricate the micro-nano sensor on the end of manipulator. We used the vibration method to detect the specific heart rates of patients, as our sensor material is graphene, the simulation and vibration frequency calculation is done for different sizes of graphene rectangular sheet. We use Ansys simulation to calculate the piezoelectric ceramic sheet vibration mode and frequency. In our simulation we find that as the size increase, the resonant frequency gradually decrease. So we choose a size of $100 \mathrm{~nm} \times 100 \mathrm{~nm}$ for the rectangular hole fabrication on the substrate surface. At last we conduct the vibration signal analysis for the certain heart rate patients, obtaining a corresponding relationship. Thus, we could do more experiments for applicable medical use.

\section{References}

[1] H. Lipkin, "A note on denavit-harterburg notation in robotics," ASME DC, 7, 2005.

[2] S.C Pradhan, A. Kumar, "Vibration Analysis of Orthotropic graphene sheets using nonlocal elasticity theory and differential quadrature method," Composite Structures, 2, pp.774-779, 2011.

[3] C.Y. Tsai, P. D. Lin, “The mathematical models of the basic entities of multi-axis series orthogonal machine tools using a modified Denavit-Hartenburg notation,"Int. J. Manuf. Technol, 42, 1016-1024, 2009.

[4] R .Ansari, S. Sahmani, B. Arash, "Nonlocal plate model for free vibrations of single-layered graphene sheets," Physics Letters A, 375, pp.53-62, 2010.

[5] B .G. Sheeparamatti, M .S Hebbal, R .B. Sheeparamatti, V .B. Math, and J .S .Kadadevaramath, "Simulation of Biosensor using FEM," Journal of Physics, 34, pp.241-246, 2006.

[6] M. S. Choi, "Free vibration analysis of plate structures using finite element-transfer stiffness coefficient method ,"KSME International Journal, 17, pp.805-815, 2003.

[7] I. Ranu, and S. C. Mohanty, "Study on Free Vibration Analysis of Rectangular Plate Structures Using Finite Element Method ,"Procedia Engineering, 38, pp.2758-2766, 2012.

[8] Z.Peng, F.Chu, and Y.He, "Vibration signal analysis and feature extraction based on reassigned wavelet scalogram ,"Journal of Sound and Vibration,5, pp.1087-1100, 2002. 
[9] F. Hu, Y. Zhang, Z. Ma, Q. Cao, Y. Xu, Z. He, and Y. Sun. A region matching method for pulse transit time estimation: improving the accuracy in determining carotid femoral pulse wave velocity, $J$ Hum Hypertension, 2015. 